\title{
Identification of Pharmacokinetic Markers for Guanxin Danshen Drop Pills in Rats by Combination of Pharmacokinetics, Systems Pharmacology, and Pharmacodynamic Assays
}

OPEN ACCESS

Edited by:

Marcello Locatelli,

Università degli Studi G. d'Annunzio

Chieti e Pescara, Italy

Reviewed by:

Gokhan Zengin,

Selçuk University, Turkey

Rufeng Wang,

Beijing University of Chinese

Medicine, China

*Correspondence:

Hong Yao

yauhung@126.com

Peiying Shi

peiyshi@126.com

${ }^{\dagger}$ These authors have contributed equally to this work

Specialty section: This article was submitted to

Ethnopharmacology,

a section of the journal

Frontiers in Pharmacology

Received: 02 September 2018 Accepted: 06 December 2018

Published: 21 December 2018

Citation:

Yao H, Huang $X$, Xie Y, Huang $X$, Ruan $Y$, Lin $X$, Huang $L$ and Shi $P$ (2018) Identification

of Pharmacokinetic Markers for Guanxin Danshen Drop Pills

in Rats by Combination

of Pharmacokinetics, Systems

Pharmacology,

and Pharmacodynamic Assays.

Front. Pharmacol. 9:1493.

doi: 10.3389/fphar.2018.01493
Hong Yao ${ }^{1 * t}$, Xiaomei Huang ${ }^{1 \dagger}$, Yunjiao Xie ${ }^{1}$, Xuliang Huang', Yijun Ruan', Xinhua Lin' ${ }^{1}$, Liying Huang ${ }^{1}$ and Peiying Shi ${ }^{2 *}$

\begin{abstract}
'Department of Pharmaceutical Analysis, School of Pharmacy, Fujian Medical University, Fuzhou, China, ${ }^{2}$ Department of Traditional Chinese Medicine Resource and Bee Products, Bee Science College, Fujian Agriculture and Forestry University, Fuzhou, China
\end{abstract}

This paper reported a feasibility study strategy of identifying pharmacokinetic (PK) markers for a cardiovascular herbal medicine, Guanxin Danshen drop pill (GDDP). First, quantification analysis revealed the constituent composition in the preparation by highperformance liquid chromatography (HPLC). Subsequently, physiochemical property calculation predicted the solubility and intestinal permeability of the constituents in the preparation. Furthermore, HPLC-MS analysis ascertained the absorbable ingredients and their PK properties in rat plasma. The main effective substances from the ingredients absorbed into blood and their cardiovascular effects were also predicted by systems pharmacology study, and were further confirmed by in vivo protective effects on isoprenaline-induced myocardial injury in mice. Finally, the ingredients with high content, representative structure feature, favorable PK properties, high relevant degree to myocardial ischemia (MI) issues, and validated therapeutic effects were considered as the PK markers for the preparation. Ginsenosides $R g_{1}, R b_{1}$, and tanshinone (TS) IIA were identified originally as PK markers for representing PK properties of GDDP. In addition, integrated PK studies were carried out according to previous reports, viz. drug concentration sum method and the AUC weighting method, to understand the in vivo process of GDDP comprehensively. The present study maybe provide a reference approach to identify PK markers for cardiovascular herbal medicines.

Keywords: Guanxin Danshen drop pills, cardiovascular herbal medicines, pharmacokinetic marker, systems pharmacology, pharmacokinetics

\section{INTRODUCTION}

Traditional Chinese medicine (TCM) recipes have been used for several thousand years in Asia. Nowadays, although Western medicine has extensively supplanted TCM in modernized cities, TCM still plays an important role in Chinese health system (Stone, 2008; Liu et al., 2009). Considerable attention has been given to the usage of herbal medicine because many TCM remedies 
show effectiveness and less adverse effect in the treatment of some diseases, in which conventional Western medicine therapies fail or are proven insufficient to provide a palliative cure (Xue and Roy, 2003; Miller and Su, 2011). Currently, only few TCM products have been approved by Western public health systems (e.g., FDA) due to the deficiency of research data from modern science experiments for the herbal medicines (Hara, 2011). Furthermore, TCM is confronted with some unfavorable critics, e.g., assailing it as an outmoded folklore (Stone, 2008). To restore local and global belief in TCMs, evidence-based investigations for TCMs (including preclinical and clinical trials) should be performed. Among the modernization efforts, pharmacokinetic (PK) study is a crucial prerequisite to make TCM products evidence-based drugs because the dosage regimens, safety and potential herb-drug interactions require reasonable estimation at the systematic exposure to them.

However, unlike Western medicine pharmaceuticals, PK investigation on TCM products is really a challenge due to the complexity of constituents (numerous unknown and known chemicals coexisting in herbal medicines) and/or lack of suitable analytical methods. Up to date, researchers are still in the struggle to grope for comprehensive and reasonable strategies or methods to measure systemic exposure to complex TCMs. Under these circumstances, PK markers were put forward to deal with the PK issues of complex TCMs. Those active constituents possessing high contents and favorable PK properties (including a significant dose-dependent systemic exposure and an appropriate elimination half-life), could be considered as PK markers for herbal products (Lu et al., 2008; Hao et al., 2009; Shi et al., 2018). As a matter of fact, researchers desire to identify those characteristic ingredients which dominate the therapeutic effects of a TCM product in vivo due to their high bioactivity and favorable systemic exposure. Identification of components that should be considered representative index (PK markers) for PK evaluation of a TCM is a matter of debate. Idealistically, the selected representative PK markers for a multicomponent TCM should combine therapeutic effects and favorable PK properties to understand the relationship between administration and effect. To date, PK investigations on TCMs with consideration of the therapeutic effects of ingredients in TCMs have been conducted (Li et al., 2015; Yang et al., 2015; Zhou et al., 2015; Chang et al., 2016; Fan et al., 2016). Nevertheless, evaluation of the therapeutic effects of all unknown and known chemicals coexisting in a TCM is impractical. Therefore, more feasible strategies for identifying the representative PK markers for multiple-component TCMs should be proposed and verified; the strategies should focus on both the favorable PK properties and potent therapeutic effects for the identified markers.

Cardiovascular disease (CVD) is the number one cause of death in the world. The World Heart Federation has reported that CVD accounts for 17.3 million deaths/year, and possibly 23.6 million deaths/year by 2030 (Smith et al., 2012; Hao et al., 2017). Because of the unmet needs for CVD control with Western medicine, clinicians is always considering the possible role of TCMs in the prevention and treatment of CVD, and a number of basic and clinical studies in this area have drawn increasing attention from the cardiovascular community (Hao et al., 2017;
Lu et al., 2018). Among the considered TCMs, Guanxin Danshen drop pill (GDDP), a compound preparation prepared using three herbs, namely, Salvia miltiorrhiza (Danshen), Panax notoginseng (Sanqi), and Dalbergia odorifera (Jiangxiang), has been widely used to prevent and treat coronary artery heart disease in clinical practice in China (Tang et al., 2016). Pharmacological studies confirmed that GDDP could increase coronary blood flow (Fang et al., 2013), decrease myocardial oxygen consumption (Cai and Dai, 2013), ameliorate hemorheology property and heart function, and alleviate ischemia reperfusion injury (Tang et al., 2016). The cardiovascular therapeutic activities of GDDP could be mainly attributed to its anti-inflammatory, antioxidation, antiplatelet aggregation, and endothelial cell protection effects (Tang et al., 2016). Ginsenosides, salvianolic acids (SAs), and tanshinones (TSs) are regarded as the main active ingredients of GDDP (Tang et al., 2016). Our previous work developed the content determination method of 17 ingredients (Figure 1), including danshensu (DSS), protocatechuic acid (PCA), protocatechuic aldehyde (PCAL), caffeic acid (CA), rosmarinic acid (RA), lithospermic acid (LA), SA B (SAB), SA A (SAA), notoginsenoside $\mathrm{R}_{1}\left(\mathrm{R}_{1}\right)$, ginsenoside $\operatorname{Rg}_{1}\left(\operatorname{Rg}_{1}\right)$, ginsenoside $\operatorname{Re}(\mathrm{Re})$, ginsenoside $R b_{1}\left(R b_{1}\right)$, ginsenoside $R d(R d)$, dihydrotanshinone I (DHTS I), cryptotanshinone (CTS), TS I (TS I), and TS IIA (TS IIA), in the preparations containing Danshen and Sanqi herbpair (Yao et al., 2017a). However, to date, there is no report on the $\mathrm{PK}$ profiles of the compound preparation. The PK properties of the active components in this preparation should be explored for clinical practice.

In this article, an approach to identify PK markers for the cardiovascular TCM preparation, GDDP, was proposed originally. The procedures and effectiveness of this method were verified and demonstrated in detail.

\section{MATERIALS AND METHODS}

\section{Chemicals and Reagents}

DSS, PCA, PCAL, CA, RA, LA, SAB, SAA, R $1, \mathrm{Rg}_{1}, \mathrm{Re}, \mathrm{Rb}_{1}$, Rd, DHTS I, CTS, TS I, TS IIA, and digoxin were purchased from Shanghai Ronghe Medicine Technology Development Co. Ltd. (Shanghai, China). The purities of all these references were greater than 98\%. Guanxin Danshen dripping pills (GDDP, batch no. YR06524) were produced by Zhongfa Industrial \& Commercial Group Yerui Pharmaceutical Co., Ltd., China according to a Chinese patent (CN200810145728.8) were purchased from a local drugstore (Fuzhou, China). Acetonitrile and methanol were of chromatographic grade (Merk KGaA, Darmstadt, Germany). Double distilled water was prepared in our lab. Glacial acetic acid was purchased from Sinopharm Chemical Reagent Co., Ltd. (Shanghai, China).

\section{Experimental Animals}

The investigation conforms to the Guide for the Care and Use of Laboratory Animals published by the US National Institutes of Health (NIH Publication No. 85-23, revised 1996). The Animal Ethic Review Committee of Fujian Medical University (Fuzhou, China) approved all procedures. Male Sprague-Dawley 


\section{Phenolic acids}

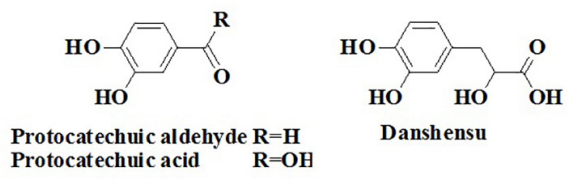<smiles>[R20]C(=O)OC(Cc1ccc(O)c(O)c1)C(=O)O</smiles><smiles>O=C(O)/C=C/c1ccc(O)c(O)c1</smiles>

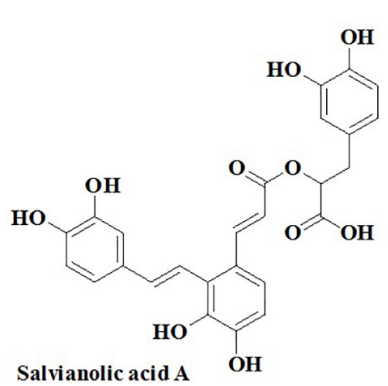<smiles>O=C(/C=C/c1ccc(O)c2c1C(C(=O)O)C(c1ccc(O)c(O)c1)O2)OC(Cc1ccc(O)c(O)c1)C(=O)O</smiles><smiles>O=C(/C=C/c1ccc(O)c2c1C(C(=O)OC(Cc1ccc(O)c(O)c1)C(=O)O)C(c1ccc(O)c(O)c1)O2)OC(Cc1ccc(O)c(O)c1)C(=O)O</smiles>

Saponins

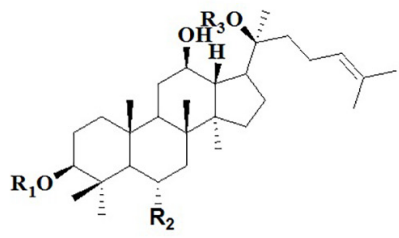

Notoginsenoside $R_{1} R_{1}=H, R_{2}=O$-GIc ${ }^{2}-X y l, R_{3}=G I c$ Ginsen oside $\mathrm{Rg}_{1} \quad \mathrm{R}_{1}=\mathbf{H}, \mathrm{R}_{2}=\mathbf{O}$-GIc, $\mathrm{R}_{3}=\mathrm{Glc}$ Ginsen oside $\mathrm{Re} \quad \mathrm{R}_{1}=\mathrm{H}, \mathrm{R}_{2}=\mathrm{O}-\mathrm{Glc}^{2}-\mathrm{Rha}, \mathrm{R}_{3}=\mathrm{Glc}$ Ginsenoside $R_{1} \quad R_{1}=G^{2} c^{2}-G I c, R_{2}=H, R_{3}=G^{-6} c^{6}-G l c$ Ginsen oside $R d \quad R_{1}=G^{2}{ }^{2}-G l c, R_{2}=H, R_{3}=G l c$<smiles>Cc1cccc2c3c(ccc12)C1=C(C(=O)C3=O)C(C)CO1</smiles>

Tanshinones
Dihydrotanshinone I

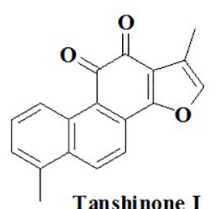<smiles>CC1COC2=C1C(=O)C(=O)c1c2ccc2c1CCCC2(C)C</smiles>

Cryptotanshinone

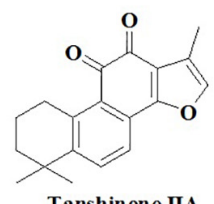

FIGURE 1 | The structures of 17 ingredients in GDDP.

rats $(200 \pm 20 \mathrm{~g})$ and Kunming mice (about 8 weeks old and 20-30 g B.W.) were obtained from Laboratory Animal Center of Fujian Medical University, Fuzhou, China) were housed in rat cages $(48 \mathrm{~cm} \times 29 \mathrm{~cm} \times 18 \mathrm{~cm})$ and mouse cages $(29 \mathrm{~cm} \times 17.8 \mathrm{~cm} \times 16 \mathrm{~cm})$, respectively, in a unidirectional airflow room under controlled temperature $\left(22 \pm 2^{\circ} \mathrm{C}\right)$, relative humidity $(40-70 \%)$, and a 12 -h light/dark cycle. Filtered tap water was available ad libitum. The mice were given commercial mouse food ad libitum. The rats were given commercial rat food ad libitum except for the overnight period before dosing. All animals were acclimated to the facilities and environment for 7 days before the experiments.

\section{Assessment of Permeability and Solubility}

Aqueous solubility (S) test of the 17 ingredients was performed by vortexing $10 \mathrm{mg}$ of each ingredient in $10 \mathrm{~mL}$ water in a test tube at ambient temperature and $1 \mathrm{~atm}$. After vortexing for $5 \mathrm{~min}$, if the solution is clear, the tested object will be considered as soluble in water. In silico assessment of the physicochemical properties governing permeation and intestinal absorption was carried out for the 17 ingredients studied. Theory aqueous solubility (S) was calculated with ALOGPS 2.1 online software via the Virtual Computational Chemistry Laboratory service (Chemoinformatics Group, Neuherberg, Germany) (VCCLAB ${ }^{1}$ ). The octanol-water partition coefficient $(\operatorname{cog} P)$, the total hydrogen bond count (donors and acceptors), and topological polar surface area (TPSA) were determined using the Molinspiration Property Calculator (Molinspiration Cheminformatics, Bratislava, Slovak Republic ${ }^{2}$ ). Lipinski's rule of 5 was used to predict the permeability of the compound (Lipinski et al., 2001).

\footnotetext{
${ }^{1}$ http://www.vcclab.org (accessed April 18, 2018)

${ }^{2}$ http:/www.molinspiration.com (accessed April 18, 2018)
} 
TABLE 1 | In vitro permeability in Caco-2 cell monolayers from literature and the results of in silico assessment of the 17 ingredients.

\begin{tabular}{|c|c|c|c|c|c|c|c|c|}
\hline \multirow[b]{2}{*}{ Analytes } & \multicolumn{2}{|c|}{$P_{\mathrm{app}}\left(\times 10^{-6} \mathrm{~cm} / \mathrm{s}\right)$} & \multirow{2}{*}{$\begin{array}{c}\text { Solubility in } \\
\text { water (mg/mL) }\end{array}$} & \multirow[b]{2}{*}{ cLogP } & \multirow[b]{2}{*}{ TPSA } & \multirow[b]{2}{*}{ MW } & \multirow[b]{2}{*}{ nON } & \multirow[b]{2}{*}{ nOHNH } \\
\hline & $\boldsymbol{P}_{\text {app (apical } \rightarrow \text { basolateral) }}$ & $\boldsymbol{P}_{\text {app (basolateral } \rightarrow \text { apical })}$ & & & & & & \\
\hline DSS & $1.22^{\mathrm{a}}$ & $0.903^{a}$ & $>10^{\mathrm{e}}$ & -0.25 & 97.98 & 198.17 & 5 & 4 \\
\hline PCA & $4.52^{b}$ & $6.91^{\mathrm{b}}$ & $>10^{\mathrm{e}}$ & 0.88 & 57.53 & 154.12 & 3 & 2 \\
\hline PCAL & $12.1^{\mathrm{a}}$ & $21.8^{a}$ & $>10^{e}$ & 0.76 & 57.53 & 138.12 & 3 & 2 \\
\hline $\mathrm{CA}$ & $4.35^{b}$ & $7.74^{b}$ & $>10^{\mathrm{e}}$ & 0.94 & 77.75 & 180.16 & 4 & 3 \\
\hline RA & $0.523^{a}$ & $0.550^{\mathrm{a}}$ & $>10^{\mathrm{e}}$ & 1.63 & 144.52 & 360.32 & 8 & 5 \\
\hline LA & $0.0816^{a}$ & $0.0413^{a}$ & $>10^{\mathrm{e}}$ & 1.57 & 211.28 & 538.46 & 12 & 7 \\
\hline SAB & $0.0200^{a}$ & $0.0377^{a}$ & $>10^{\mathrm{e}}$ & 1.61 & 278.04 & 718.62 & 16 & 9 \\
\hline SAA & $0.0595^{a}$ & $0.0554^{a}$ & $>10^{e}$ & 3.01 & 184.97 & 494.45 & 10 & 7 \\
\hline $\mathrm{R}_{1}$ & $>0.1^{c}$ & $>0.1^{c}$ & $>10^{e}$ & 1.49 & 298.14 & 933.14 & 18 & 12 \\
\hline $\mathrm{Rg}_{1}$ & $>0.1^{c}$ & $>0.1^{c}$ & $>10^{e}$ & 2.77 & 239.22 & 801.02 & 14 & 10 \\
\hline $\mathrm{Re}$ & $>0.1^{c}$ & $>0.1^{\mathrm{c}}$ & $>10^{e}$ & 2.07 & 298.14 & 947.17 & 18 & 12 \\
\hline $\mathrm{Rb}_{1}$ & $>0.1^{c}$ & $>0.1^{c}$ & $>10^{e}$ & 0.28 & 377.29 & 1109.31 & 23 & 15 \\
\hline $\mathrm{Rd}$ & $>0.1^{c}$ & $>0.1^{\mathrm{c}}$ & $>10^{e}$ & 1.98 & 298.14 & 947.17 & 18 & 12 \\
\hline DHTS I & $1.49^{d}$ & $8.28^{d}$ & 0.0077 & 3.51 & 43.38 & 278.31 & 3 & 0 \\
\hline CTS & $1.59^{d}$ & $8.36^{d}$ & 0.0098 & 3.83 & 43.38 & 296.37 & 3 & 0 \\
\hline TS I & $0.95^{d}$ & $11.27^{d}$ & 0.0115 & 3.83 & 47.28 & 276.29 & 3 & 0 \\
\hline TS $\| A$ & $0.98^{d}$ & $11.81^{d}$ & 0.0104 & 4.16 & 47.28 & 294.35 & 3 & 0 \\
\hline
\end{tabular}

a-d Mean that the data were collected from the previous reports by Lu et al. (2008), Bowles et al. (2017), Liu et al. (2009), and Hu et al. (2014), respectively.

e The values were experiment data in water at ambient temperature and $1 \mathrm{~atm}$.

In addition, the bi-directional transport assay in Caco-2 cells for the mentioned ingredients in this paper has ever been carried out to evaluate their gastrointestinal absorption properties, and the reported bidirectional $P_{\text {app }}$ [including $P_{\text {app(basolateral } \rightarrow \text { apical) }}$ and $\left.P_{\text {app(apical } \rightarrow \text { basolateral })}\right]$ and efflux ratio $\left[P_{\text {app (basolateral } \rightarrow \text { apical })} / P_{\text {app (apical } \rightarrow \text { basolateral })}\right]$ values were listed in Table 1.

\section{Administration and Sample Pretreatment for Multiple Component PK Study}

Six rats were classified randomly into two groups, received a single p.o. administration of GDDP at 0.4 and $0.8 \mathrm{~g} / \mathrm{kg}$ doses (the dosing solutions were prepared unifiedly into $1 \mathrm{~mL}$ with physiologic saline), respectively. Approximately $300 \mu \mathrm{L}$ of blood was collected from the cut-tail before dosing and at the following time points 10, 15, 30, 45, $60 \mathrm{~min}$, and 2, 4, 6, 8, 12, and $24 \mathrm{~h}$ after dosing. Plasma was isolated from the blood samples by centrifugation and then stored at $-20^{\circ} \mathrm{C}$ until analysis.

For LC-MS analysis, samples stored were allowed to thaw at ambient temperature, followed by shaking for $30 \mathrm{~s}$ via a vortex apparatus. Plasma $(100 \mu \mathrm{L})$ was removed and transferred to an appropriately labeled polypropylene tube $(1.5 \mathrm{~mL})$ containing $10 \mu \mathrm{L}$ internal standard solution (5 $\mu \mathrm{g} / \mathrm{mL}$ digoxin) and methanol $(300 \mu \mathrm{L})$, followed by shaking for 2 min using a vortex apparatus. The samples were centrifuged $(15,000 \mathrm{rpm}$ for $10 \mathrm{~min}$ at $4^{\circ} \mathrm{C}$ ) and $2 \mu \mathrm{L}$ clear supernatant was injected into HPLC-MS system for bioanalysis by a validated HPLC-MS method.

\section{LC/MS Bioassays}

Selection ion monitoring (SIM) and multiple reaction monitoring (MRM) LC-MS method were developed simultaneously, and used to determine saponins and TSs in rat plasma, respectively.

The apparatus used was a Shimadazu LC 20A system consisting of a binary pump, a degasser, an auto-sampler, and a thermostat. Separation was carried out by elution on a Ultimate XB-C18 column $(50 \mathrm{~mm} \times 4.6 \mathrm{~mm}, 3.5 \mu \mathrm{m})$. The mobile phase consisted of deionized water-acetic acid (A; 100:0.5, v/v) and acetonitrile (B). The gradient elution was employed as follows: $30-50 \% \mathrm{~B}$ at $0-2 \mathrm{~min} ; 50-80 \% \mathrm{~B}$ at $2-3 \mathrm{~min} ; 80-90 \% \mathrm{~B}$ at 3-4 $\mathrm{min} ; 90 \% \mathrm{~B}$ at $4-9 \mathrm{~min} ; 95 \% \mathrm{~B}$ at $9.1-11 \mathrm{~min}$. The reequilibrium took $9 \mathrm{~min}$, giving a total run time of $20 \mathrm{~min}$. The flow rate was $0.6 \mathrm{~mL} / \mathrm{min}$. The column temperature kept at $30^{\circ} \mathrm{C}$ and the volume of sample injected was $2 \mu \mathrm{L}$.

Mass spectrometric detection was carried out with a triple quadrupole mass spectrometer (LCMS 8040; Shimadazu Corporation, Japan), operating in the negative electrospray ionization (ESI) mode for saponins and positive ESI mode for TSs. ESI and collision energy were optimized to maximize generation of the characteristic adduct ions, or product ions, respectively. The SIM ions for $\mathrm{R}_{1}, \mathrm{Rg}_{1}, \mathrm{Rb}_{1}, \mathrm{Rd}$, and digoxin were $m / z 931.35,859.4,1107.4,1005.4$, and 779.6, respectively, in negative ionization mode. The precursor-to-product ion pairs used for MRM of DHTS I, CTS, TS I, and TS IIA were $m / z$ $279 \rightarrow 261,297 \rightarrow 251,277 \rightarrow 249$, and $295 \rightarrow 277$ in positive ionization mode, respectively, and digoxin was at $\mathrm{m} / z 839 \rightarrow 649$ in negative ionization mode.

The HPLC-MS method was validated by linearity, limit of quantification (LOQ), limit of detection (LOD), precision, accuracy, extraction recovery, matrix effect, and stability for the eight ingredients, $\mathrm{R}_{1}, \mathrm{Rg}_{1}, \mathrm{Rb}_{1}, \mathrm{Rd}$, DHTS I, CTS, TS I, and TS IIA in plasma. Calibration curves were plotted for the 
eight ingredients using weighted linear regression of the ratio of analyte and internal standard (digoxin, $500 \mathrm{ng} / \mathrm{mL}$ ) peak areas against the corresponding nominal concentration of the analyte. LOQ was defined as the lowest concentration at which both precision and accuracy were below 20\% with the ratio of signal and noise more than $10(S / N>10)$. LOD was defined as the detectable concentration at which the ratio of signal and noise was more than $3(S / N>3)$. Intra- and inter-day accuracy and precision were assessed by detecting of QC samples using six replicates of rat samples at three concentration levels for all the eight ingredients SAA (2, 400, and $1600 \mathrm{ng} / \mathrm{mL}$ ) on one or three validation days. Accuracy and precision were expressed by relative error (RE) and coefficient of variance (RSD), respectively. The extraction recovery, matrix effect, and stability were determined by the QC samples above-mentioned according to previous reports (Shi et al., 2015; Yang et al., 2016).

\section{Systems Pharmacology Studies}

Firstly, the 3D structures of the ingredients identified with favorable PK properties were drawn, operated by energy minimization (MM2 force fields) and saved as "mol2" type files with Chem 3D Ultra 10.0 software (CambridgeSoftware). All the mol2 type files were submitted to PharmMapper Server (a freely accessed web-server for target fishing, available online at http:// lilab.ecust.edu.cn/pharmmapper/index.php) (accessed on March 10, 2018) for searching potential target candidates from 2241 human protein targets for the given small molecules (Liu et al., 2010; Wang et al., 2016; Wang et al., 2017). As a result, predicted top 100 targets ranked by normalized fit score in descending order were obtained from the PharmMapper. Among the targets, those with positive $z^{\prime}$-score and indicated CVDs relevance were identified as the potential target proteins of the corresponding ingredients, and remained for the following operations. Next, searching the Uniprot databases ${ }^{3}$ to obtain the UniProtKB identifier of each potential target protein and sending the UniProtKB identifiers to MAS 3 (molecule annotation system ${ }^{4}$ ) to query for the possible CVDs. The resulting proteins were used to construct the C-T-D network graphs using Cytoscape software. ${ }^{5}$ Meanwhile, the target fishing fit scores and correlation degree of target protein in the pharmacology networks corresponding to each ingredient were used to evaluate their possible effect contribution against myocardial ischemia (MI) diseases.

\section{In vivo Anti-MI Study in Mice}

The tests were carried out according to our previous report (Yao et al., 2017b) with slight modification. Briefly, mice were randomly allocated into eleven groups $(n=8)$. The first two groups received physiologic saline $(0.2 \mathrm{~mL}$, p.o.) and a selfprepared solution (40\% PEG-400: 5\% Tween-80: water $=$ 54:1:45, $0.2 \mathrm{~mL}$, p.o.) for 7 days and served as sham and model groups, respectively. The remaining nine groups received GDDP, $R_{1}$, $\mathrm{Rg}_{1}, \mathrm{Rb}_{1}, \mathrm{Rd}$, DHTS I, CTS, TS I, and TS IIA, respectively (administration solutions were prepared with the self-prepared

\footnotetext{
${ }^{3}$ http://www.uniprot.org/uniprot/

${ }^{4}$ http://bioinfo.capitalbio.com/mas3/

${ }^{5}$ http://www.cytoscape.org/
}

solution; $0.8 \mathrm{~g} / \mathrm{kg}$ p.o. dose for GDDP, and $40 \mathrm{mg} / \mathrm{kg}$ p.o. dose for others) for 7 days. All groups, except for the sham one, received $5 \mathrm{mg} / \mathrm{kg}$ isoprenaline chloride once daily for two successive days (in the fourth and fifth days of treatment). Thirty minutes after administration on the fifth and seventh days of the test, animals were anesthetized with pentobarbital sodium (70 mg/kg; i.p.) for ECG monitoring with standard artifact free lead II (right forelimb to left hind limb). Needle electrodes were inserted subcutaneously into limbs of each mouse and connected to MD3000 bioinformation collector (Huaibei Zhenghua Bioinstrument Co., Ltd., Anhui, China). Twenty-four hours after the last administration, mice were then sacrificed by decapitation. Immediately after the sacrifice of the mice, the hearts treated for sectioning and hematoxylin and eosin (H\&E) staining. Sections from the left ventricle were examined by light microscopy (Leica DMR, Germany) at $200 \times$ magnification.

\section{Data Processing}

Non-compartmental PK parameters were calculated by DAS 2.0 software (Chinese Pharmacologic Society, Beijing, China). For plasma $\mathrm{PK}$, the area under the plasma concentration-time curve from time zero to the last measurable concentration $\left(\mathrm{AUC}_{0 \rightarrow \mathrm{t}}\right)$ was calculated using trapezoidal rule, the $\mathrm{AUC}_{0 \rightarrow \infty}$ was obtained by extrapolating $\left(\mathrm{AUC}_{0 \rightarrow \mathrm{t}}\right)$ to infinity, the apparent elimination half-life $\left(t_{1 / 2} \beta\right)$ was calculated from the terminal loglinear portion of plasma, and the total body clearance $\left(\mathrm{Cl}_{\text {tot }}\right)$, apparent volume of distribution $\left(\mathrm{V}_{\mathrm{d}}\right)$, mean residence time (MRT), etc. were also calculated by non-compartmental PK mode.

For further comparison by the plasma drug concentration sum method, the plasma drug concentrations of the three markers at each PK time point were summed directly to obtain the proximately total markers concentration in plasma. The resulted total concentration-time curve was prepared by plotting the PK time point against the relative sum concentration. In addition, AUC weighting integrated analysis for the three markers was performed according to the previous reports (Lu et al., 2008; Hao et al., 2009; Yao et al., 2017b).

All the results are expressed as mean \pm S.D. A single-tailed Student's $t$-test was performed in the work.

\section{RESULTS}

\section{Strategy and Principles for Identifying PK Markers}

Before exploring the PK of a TCM, its constituents should be identified and determined, so that the ingredient contents could be sorted and the structure classification of the ingredients could be further discussed. During experiments, the identified PK markers of a TCM should exhibit favorable drug-like properties (Lu et al., 2008; Shi et al., 2018), such as appropriate PK properties (due to suitable solubility in water and intestinal permeability) and desired bioactivities. The selected PK markers should possess appropriate PK properties to suggest that the bioactive PK markers could pass through biological barrier to distribute toward action sites with therapeutic concentration 
levels after administration of the TCM. Possessing the desired bioactivities is crucial so that the identified PK markers can at least partly reflect the pharmacological effect of the TCM. Meanwhile, systems pharmacology could conveniently dissect the therapeutic effects and mechanisms of multiple components of complex systems (Danhof, 2016), such as herbal medicines or TCMs (Zhang et al., 2015; Zheng et al., 2016). Especially, the connection degree of a certain disease (e.g., MI referring to myocardial infarction, coronary ischemic syndrome, coronary heart disease, angina, ischemia, heart failure, oxidative injury, etc.) in a constructed compounds-targets-diseases (C-T-D) network could be considered as an loose reflection of effect contribution of an ingredient to a certain disease. The fit scores of proteins in target fishing by PharmMapper and the correlation degree of target proteins in C-T-D network could also be considered as a loose index to represent the target proteins' importance against the certain disease. Therefore, systems pharmacology seems to be able to predict the effect substances base of a TCM against a certain disease, which could also provide important information for understanding or evaluating the therapeutic effect of multiple components of the TCM.

Considering all the relevant factors, a strategy for identifying PK markers was proposed. As shown in Figure 2, the six main steps are as follows: (1) determining the component constituents and quantifying the chemical ingredients of the studied TCM; (2) sorting and categorizing the ingredients by their contents size and parent structures (e.g., phenolic acids, saponins, and TSs) to determine the ingredients with high content and representative structure feature; (3) in silico assessment of the physiochemical properties of the ingredients, including octanol-water partition coefficient $(\log \mathrm{P})$, solubility in water (S), topological molecular polar surface area (TPSA), number of hydrogen bond acceptors ( $\mathrm{nON}$ ), and number of hydrogen bond donors ( $\mathrm{nOHNH}$ ), to predict the intestinal permeability and provide reference information for subsequent study; (4) PK profiling of the ingredients absorbed into blood after administration of the TCM to rats by high sensitive and selective analysis methods, such as high-performance liquid chromatography-triple quadrupole mass spectrometry (HPLCMS/MS); (5) predicting the effect contribution of the ingredients absorbed into blood against MI by systems pharmacology methods and further ascertaining the cardiovascular effects on disease animal models to identify the main effective ingredients in the TCM; and (6) considering the results from the in silico assessment, PKs, systems pharmacology, and pharmacological tests, the ingredients with high content, representative structure feature, favorable PK properties (suitable systematic exposure and drawable PK curves), high cardiovascular effect relevant degree, and validated therapeutic effects could be considered the PK markers for the preparation.

\section{Chemical Components in GDDP}

In our previous research (Yao et al., 2017a), an HPLC method was developed to determine the chemicals and their contents in TCM compound preparations containing Danshen-Sanqi herb pair. A total of 17 ingredients were determined in GDDP
(Figure 1). According to their structure characterization, these ingredients could be classified into three categorization, viz. phenolic acids (including DSS, PCA, PCAL, CA, RA, LA, SAB, and SAA), saponins (including protopanaxatriols $\mathrm{R}_{1}, \mathrm{Rg}_{1}$, and Re and protopanaxatriols $\mathrm{Rb}_{1}$ and $\mathrm{Rd}$ ), and TSs (DHTS I, CTS, TS I, and TS IIA). Among them, Rd (15,146.80 $\pm 1.92 \mu \mathrm{g} / \mathrm{g})$, $\mathrm{Rg}_{1}(6119.50 \pm 3.06 \mu \mathrm{g} / \mathrm{g}), \mathrm{Rb}_{1}(4040.30 \pm 1.84 \mu \mathrm{g} / \mathrm{g})$, TS IIA (3212.17 $\pm 2.31 \mu \mathrm{g} / \mathrm{g}), \mathrm{R}_{1}(1221.00 \pm 0.38 \mu \mathrm{g} / \mathrm{g})$, and $\mathrm{SAB}(1045.00 \pm 0.32 \mu \mathrm{g} / \mathrm{g})$ were higher than others (beyond $0.1 \%$ in the preparation). The contents of the remaining ingredients were lower than $0.07 \%$; especially, the PCA $(26.40 \pm 0.18 \mu \mathrm{g} / \mathrm{g})$, PCAL $(12.30 \pm 0.03 \mu \mathrm{g} / \mathrm{g})$, CA $(50.9 \pm 0.04 \mu \mathrm{g} / \mathrm{g})$, and DHTS I $(56.6 \pm 0.02 \mu \mathrm{g} / \mathrm{g})$ contents were considerably low (below $0.01 \%$ ). The content ordering was as follows: $\mathrm{Rd}>\mathrm{Rg}_{1}>\mathrm{Rb}_{1}>$ TS IIA $>\mathrm{R}_{1}>\mathrm{SAB}>\mathrm{CTS}>\mathrm{TS}$ $\mathrm{I}>\mathrm{RA}>\mathrm{LA}>\mathrm{DSS}>\mathrm{Re}>\mathrm{SAA}>$ DHTS $\mathrm{I}>\mathrm{CA}>\mathrm{PCA}>$ PCAL $(\mathrm{P}<0.05$, each ingredient vs. the others). The phenolic acids, saponins, and TSs were approximately 2.41, 26.77, and $4.42 \mathrm{mg} / \mathrm{g}$, respectively. In addition, GDDP also included a major pharmaceutic adjuvant PEG 6000 (occupying about 70\% mass in the preparation) and volatile oil from Jiangxiang. According to the results and considering the low oral administration dosage for human beings (e.g., $0.4 \mathrm{~g}$ per administration), the ingredients absorbed into blood could be mainly saponins.

\section{Molecular Physiochemical Properties}

As listed in Table 1, the determined solubility (S) of the eight phenolic acids and five saponins were more than $10 \mathrm{mg} / \mathrm{mL}$ in water at $25{ }^{\circ} \mathrm{C}$ at $1 \mathrm{~atm}$. However, DHTS I, CTS, TS I, and TS IIA, were insoluble in water at $25^{\circ} \mathrm{C}$, and their calculated $\mathrm{S}$ values were $7.7,9.8,11.5$, and $10.4 \mu \mathrm{g} / \mathrm{mL}$, respectively. These values suggested their low plasma drug concentrations and poor bioavailability. The calculated $\log P(c \log P)$ values for the four TS ingredients were more than 3 , which indicated their high hydrophobicity. The cLogP values for PCA, PCAL, CA, RA, SAA, $\mathrm{SAB}, \mathrm{Rb}_{1} \mathrm{R}_{1}, \mathrm{Rg}_{1}, \mathrm{Re}$, and $\mathrm{Rd}$ were between 0 and 3, thereby suggesting their moderate hydrophobicity and hydrophilicity. Moreover, only DSS, PCA, PCAL, CA, DHTS I, CTS, TS I, and TS IIA were favorable on all the values of cLogP, TPSA, MW, $\mathrm{nON}$, and $\mathrm{nOHNH}$ according to the Lipinski's rule of 5 (favorable $\log \mathrm{P}<5$, TPSA $<140, \mathrm{MW}<500, \mathrm{nON} \leq 10$, and $\mathrm{nOHNH} \leq 5)$ (Lipinski et al., 2001). These results possibly indicated their favorable intestinal permeability and drug-likeness. In fact, the in vitro permeability for all the 17 ingredients have ever been reported using Caco-2 permeability assay (Lu et al., 2008; Liu et al., 2009; Hu et al., 2014; Bowles et al., 2017). According to the previous results (Table 1), also only DSS, PCA, PCAL, CA, DHTS I, CTS, TS I, and TS IIA showed the apparent permeability of $\geq 10^{-6} \mathrm{~cm} / \mathrm{s}$ in Caco- 2 cell monolayers and were considered to be favorable for their absorption in vivo. However, considering the low solubility or considerably low contents, the plasma concentrations of phenolic acids and TSs could also be low after oral administration of GDDP in rats. Meanwhile, $\mathrm{R}_{1}, \mathrm{Rg}_{1}, \mathrm{Rb}_{1}$, and $\mathrm{Rd}$, possessed moderate apparent permeability $\left(>10^{-7} \mathrm{~cm} / \mathrm{s}\right.$ ) (Liu et al., 2009), high solubility and contents, suggesting that they could be detected relatively easy in blood after oral administration of the GDDP. These 


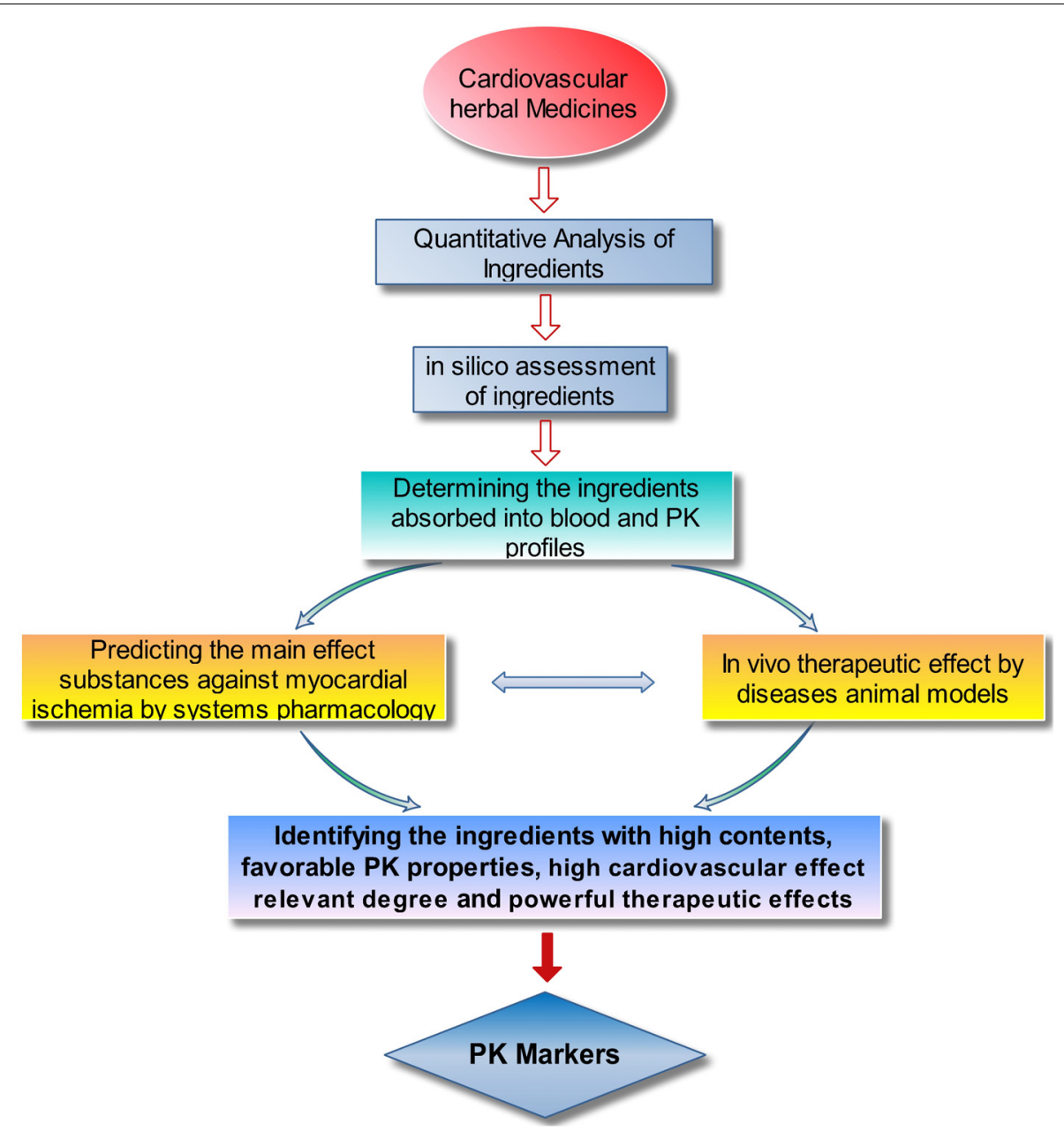

FIGURE 2 | The proposed strategy for identifying PK markers for cardiovascular TCMs.

results provided useful reference information for subsequent procedures, such as identifying the absorbable ingredients in plasma and understanding their low systematic exposure levels.

\section{Multiple-Component PK Profiles for GDDP}

Taking the 17 ingredients quantified in GDDP as the observed objects, we identified those absorbed into blood by HPLCMS/MS with SIM or MRM modes after oral administration of GDDP to rats with a dosage of $0.8 \mathrm{~g} / \mathrm{kg} \mathrm{B.W}$. (equal to 6.7 times dosage per day to human beings). Thirteen ingredients, including DSS, PCAL, PCA, CA, $\mathrm{R}_{1}, \mathrm{Rg}_{1}, \mathrm{Re}, \mathrm{Rb}_{1}$, Rd, DHTS I, CTS, TS I, and TS IIA, could be detected (LOD, $0.5-0.7 \mathrm{ng} / \mathrm{mL}$ ) at $5,10,15$, 30,45 , or 60 min time points in rat plasma after administration. However, among them, only $\mathrm{R}_{1}, \mathrm{Rg}_{1}, \mathrm{Rb}_{1}$, Rd, DHTS I, CTS, TS I, and TS IIA could be determined (LOQ, $1.0-1.4 \mathrm{ng} / \mathrm{mL}$ ) at the planned successive PK time points $(5,10,15,30,45,60$, and $120 \mathrm{~min}$ ). Furthermore, the plasma levels of saponins were more than10-fold higher than those of the TSs. These results agreed with the predictions from the in silico assessment and the ingredients' content size.

For the PK analysis of $\mathrm{R}_{1}, \mathrm{Rg}_{1}, \mathrm{Rb}_{1}$, Rd, DHTS I, CTS, TS I, and TS IIA in rats, the methodological validation referring to specificity, linearity, LOD, LOQ, precision, accuracy, extraction recovery, matrix effects, and stability, was performed. The typical SIM chromatograms for $\mathrm{R}_{1}, \mathrm{Rg}_{1}, \mathrm{Rb}_{1}$ and $\mathrm{Rd}$ and MRM chromatograms for DHTS I, CTS, TS I, and TS IIA are shown in Supplementary Figures S1 and S2, respectively. No endogenous interference could be observed, which suggested the good specificity of the presented HPLCMS bioanalysis. Supplementary Table S1 provides a list of the calibration parameters, namely, regression equation, linearity range, $R^{2}$, LOD, and LOQ, for the eight ingredients. Results showed good linearity $\left(R^{2}>0.99\right)$, wide range (about $1-2600 \mathrm{ng} / \mathrm{mL})$, and excellent LOQs $(<1.4 \mathrm{ng} / \mathrm{mL})$ and LODs $(<0.7 \mathrm{ng} / \mathrm{mL})$. Supplementary Table S2 lists the results of precision (RSDs $<20 \%$ ) and accuracy (REs, -18.22 to $-12.99 \%$ ). Results of extraction recoveries and matrix effects are presented in Supplementary Table S3. The sample stabilities after $24 \mathrm{~h}$ in autosampler vials, three freeze-thaw cycles, and placing plasma 

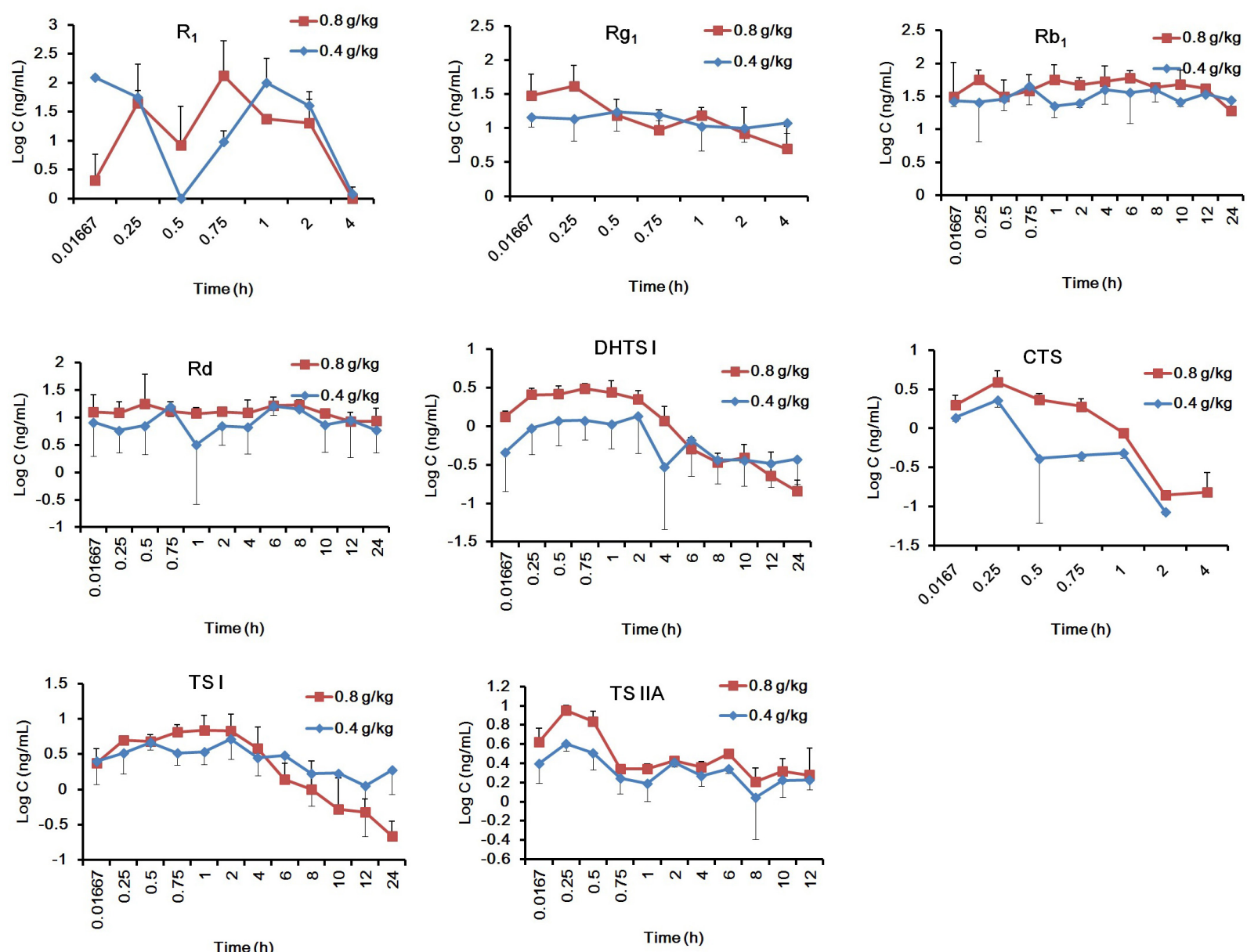

FIGURE 3 | The mean plasma drug concentration-time profiles of the eight ingredients after oral administration of GDDP to rats with the dosages 0.4 , and $0.8 \mathrm{~g} / \mathrm{kg}$ B.W.

sample for $6 \mathrm{~h}$ at $4^{\circ} \mathrm{C}$ are shown in Supplementary Table S4. These results demonstrated that the method was available for simultaneous determination of the eight ingredients in rat plasma.

The validated method was successfully applied to the PK study of $\mathrm{R}_{1}, \mathrm{Rg}_{1}, \mathrm{Rb}_{1}, \mathrm{Rd}$, DHTS I, CTS, TS I, and TS IIA in rat plasma after intragastric administration of GDDP. The mean plasma drug concentration-time profiles of the ingredients after oral administration of GDDP $(0.4$ and $0.8 \mathrm{~g} / \mathrm{kg})$ to rats are shown in Figure 3.

The main PK parameters were obtained from the noncompartmental mode calculation. As listed in Supplementary Table S5, the systematic exposure to $\mathrm{Rb}_{1}$ was the largest, that is, 3.5-30 times higher than those of $\mathrm{Rd}, \mathrm{R}_{1}, \mathrm{Rg}_{1}$, TS IIA, and TS I $(P<0.05)$, and 84-1137 times higher than those of CTS and DHTS $(P<0.05)$, according to the $\mathrm{AUC}_{0-\infty}$. The order of the ingredients based on the $\mathrm{AUC}_{0-\infty}$ was $\mathrm{Rb}_{1}>\mathrm{Rd}>\mathrm{R}_{1}>\mathrm{TS}$ IIA $\geq \operatorname{Rg}_{1} \geq$ TS I $>$ DHTS I $>$ CTS. According to the $t_{1 / 2}$ $\beta$, the ingredients could be classified into two categorizations, namely, fast elimination type $\left(t_{1 / 2} \beta<2 \mathrm{~h}\right)$ including $\mathrm{R}_{1}, \mathrm{Rg}_{1}$, and CTS, and slow elimination type $\left(t_{1 / 2} \beta>2.6 \mathrm{~h}\right)$, including
$\mathrm{Rb}_{1}, \mathrm{Rd}$, TS I, DHTS I, and TS IIA. The order of $\mathrm{MRT}_{0-\mathrm{t}}$ and $\mathrm{MRT}_{0-\infty}$ values further supported the classification according to the $t_{1 / 2} \beta$ values. In general, the PK behavior of $\mathrm{Rg}_{1}$ was similar to those of other protopanaxatriols and that of $\mathrm{Rb}_{1}$ was similar to those of other protopanaxadiols. In addition, according to the $C_{\max }$ values, the ingredients could be classified into two categorizations: with relatively high plasma drug concentrations, including $\mathrm{R}_{1}, \mathrm{Rb}_{1}, \mathrm{Rd}$, and $\mathrm{Rg}_{1}\left(C_{\max }>20 \mathrm{ng} / \mathrm{mL}\right)$, and with low plasma drug concentrations, including TS I, DHTS I, TS IIA, and CTS $\left(C_{\max }<10 \mathrm{ng} / \mathrm{mL}\right)$. According to these results from the PK studies, the $\mathrm{PK}$ properties of $\mathrm{R}_{1}, \mathrm{Rb}_{1}, \mathrm{Rd}$, and $\mathrm{Rg}_{1}$ could be more favorable (e.g., easily determined plasma concentration levels and larger AUCs) than those of the TSs.

\section{Systems Pharmacology Analysis}

During the systems pharmacology investigation, querying MAS 3 (molecule annotation system, see text footnote 4) obtained some potential target proteins which were explicitly marked to be correlated to cardiovascular issues. Among of them, prothrombin (F2, Uniprot ID: P00734), mitogen-activated protein kinase 10 (MAPK10, Uniprot ID: P53779), estrogen receptor (ESR1, 
Uniprot ID: P03372), caspase-3 (CASP3, Uniprot ID: P42574), chymase (CMA1, Uniprot ID: P23946), amine oxidase B (MAOB, Uniprot ID: P27338), and superoxide dismutase 2 (SOD2, Uniprot ID: P04179) were closely relative to $\mathrm{MI}$ issues inferring to myocardial infarction, coronary ischemic syndrome, coronary heart disease, angina, ischemia, heart failure, or oxidative injury. As shown in Figure 4, the eight ingredients, $\mathrm{Rg}_{1}, \mathrm{Rb}_{1}, \mathrm{Rd}, \mathrm{R}_{1}$, TS IIA, DHTS I, CTS, and TS I with favorable PK properties show different therapeutic relevance to the MI issues. The connection frequency (relevance degree) on the MI issues for the eight ingredients above are $8,7,6,5,6,4,3$, and 3 , respectively, in the C-T-D networks. The loose relationship between the ingredients and the MI diseases inspired us to predict that the therapeutic effects for $\mathrm{Rg}_{1}$ and $\mathrm{Rb}_{1}, \mathrm{Rd}$, TS IIA on MI diseases could be more powerful than those for $\mathrm{R}_{1}$, DHTS I, CTS, and TS I.

For further analysis, the fit scores of proteins to the above eight ingredients for target fishing by PharmMapper and the correlation degree of target proteins in the C-T-D networks also listed in Table 2. According to the results, three potential target proteins (closely relative to MI issues), F2, MAPK10, and ESR1 showed high correlation degree in the C-T-D networks. Accordingly, their total values of correlation degree in the C-T-D networks are relatively large $(60,62$, and 46 , respectively). The sum of fit scores in target fishing by PharmMapper for the studied eight ingredients as to F2, MAPK10, or ESR1 are also relatively high $(14.587,19.86$, or 20.226 , respectively). The results suggested that the three proteins could be the important targets for the multiple ingredients of GDDP against MI issues. Meanwhile, as to the individual ingredient, the sums of correlation degree/fit scores of the potential targets closely relative to $\mathrm{MI}$ issues in the $\mathrm{C}-\mathrm{T}-\mathrm{D}$ networks were $36 / 16.529$, 35/11.855, 37/11.754, 29/11.771, 29/8.174, 26/8.308, 14/5.618, and $17 / 5.618$ for $\mathrm{Rg}_{1}, \mathrm{Rb}_{1}, \mathrm{Rd}, \mathrm{R}_{1}$, TS IIA, DHTS I, CTS, and TS I, respectively. These results further encouraged us to predict that $\mathrm{Rg}_{1}, \mathrm{Rb}_{1}$, and $\mathrm{Rd}$ could be the most important therapeutic ingredients to MI diseases, and TS IIA was more powerful than the others three TSs, DHTS I, CTS, and TS I in the GDDP.

\section{Protective Effects of the Ingredients on Isoprenaline-Induced MI in Mice}

$\mathrm{R}_{1}, \mathrm{Rb}_{1}, \mathrm{Rd}, \mathrm{Rg}_{1}$, TS I, DHTS I, TS IIA, and CTS were further examined (40 mg/kg dosage for each; p.o. dosing) in the anti-MI study in mice. As shown in Figure 5A, the ECG of the normal mice showed regular pattern with defined P, QRS, and $\mathrm{T}$ waves. Isoprenaline-induced MI mouse model showed abnormal QRS wave and reversed $\mathrm{T}$ wave in $30 \mathrm{~min}$ after the second injection of isoprenaline to mice (on the fifth day of the trial) and significantly elevated ST segments $(P<0.05$ vs. sham group) on the seventh day of the trial (Figure 5B). These observations indicated the MI mouse models were prepared successfully. The abnormity of QRS waves occurred in all the eight ingredient-treated groups after modeling; additionally, $\mathrm{T}$ waves reversed or decreased in the $\mathrm{R}_{1}, \mathrm{Rg}_{1}, \mathrm{Rb}_{1}$, DHTS I, CTS, and TS I groups (Figures $\mathbf{5} \mathbf{C}-\mathbf{H}$ ), except for Rd- and TS
IIA-treated groups on the fifth day of the trial (ST segment elevated) (Figures 5I,J). As shown in Supplementary Figure S3, the ST segment elevations in $\mathrm{Rg}_{1}$-and TS IIA-treated groups were significantly decreased compared with that in model group $(P<0.05$, vs. MI group) on the seventh day of the trial.

Histopathology of mouse heart from control group showed a normal architecture structure with striations, branched appearance, fusiform shape, and continuity with adjacent myofibrils (Figure 6A). Heart tissue from the MI mouse models showed apparent cell distortion, edema, hypochromatosis, and inferior continuity with adjacent myofibrils (Figure 6B). Tissue sections from the $\mathrm{Rg}_{1}$ - and $\mathrm{Rb}_{1}$-treated groups showed less severe histological damage, viz. slight cell distortion, edema, and good continuity with adjacent myofibrils (Figures 6D,E). TS IIA-treated group showed edema, inflammatory infiltration, and loss of striations in heart tissue (Figure 6J). The TS I-treated group showed myocardial lysis, architecture disorder, and loss of striations in heart tissue (Figure 6I). Nevertheless, all the CTS-, DHTS I-, Rd-, and $\mathrm{R}_{1}$-treated (Figures 6H,G,F,C, respectively) groups exhibited remarkably severe histological damage with extensive myocardial lysis and architecture wrecking. These results suggested that $\mathrm{Rg}_{1}$ and $\mathrm{Rb}_{1}$ possessed favorable anti-MI effect, and TS IIA could also exert mild therapeutic effect against CVDs in GDDP.

\section{Identifying PK Markers and Integrated PK Investigation for GDDP}

Chemical component analysis results pointed out that $\mathrm{Rd}$, $\mathrm{Rg}_{1}, \mathrm{Rb}_{1}$, TS IIA, $\mathrm{R}_{1}$, and $\mathrm{SAB}$ were the main components in GDDP. Assessment of molecular physiochemical properties showed that $\mathrm{R}_{1}, \mathrm{Rg}_{1}, \mathrm{Rb}_{1}$, and $\mathrm{Rd}$ could be detected relatively easy in blood after oral administration of the GDDP. The multiple-component $\mathrm{PK}$ studies further confirmed that $\mathrm{R}_{1}, \mathrm{Rg}_{1}$, $\mathrm{Rb}_{1}$, and $\mathrm{Rd}$ possessed relatively more favorable $\mathrm{PK}$ properties than those of other ingredients in GDDP, although the TSs, DHTS I, CTS, TS I, and TS IIA could also be determined at the studied PK time points. Systems pharmacology deduced $\operatorname{Rg}_{1}$, $\mathrm{Rb}_{1}$, and $\mathrm{Rd}$ could be the important therapeutic ingredients to MI diseases and TS IIA could also be the most important one of active TSs absorbable into circulation system, and they should be further weighed and considered. Evidence from the in vivo anti-MI experiments confirmed that only $\mathrm{Rg}_{1}$ and $\mathrm{Rb}_{1}$ were the most effective components, and TS IIA exhibited mild positive effect against MI injury in MI model mice. Finally, comprehensively considering the content sizes, molecular physiochemical properties, PK characterizations, and therapeutic effects, $\mathrm{Rg}_{1}, \mathrm{Rb}_{1}$, and TS IIA were identified as the PK markers for profiling the in vivo process of protopanaxatriols, protopanaxadiols, and TSs, respectively, in GDDP. These three ingredients displayed high content, representative structure feature (protopanaxatriols, protopanaxadiols, and TSs), favorable PK properties, and validated therapeutic effects.

To obtain easy PK parameters, we proposed to carry out the integrated PK studies by using the identified PK markers according to previous reports, viz. drug concentration sum 

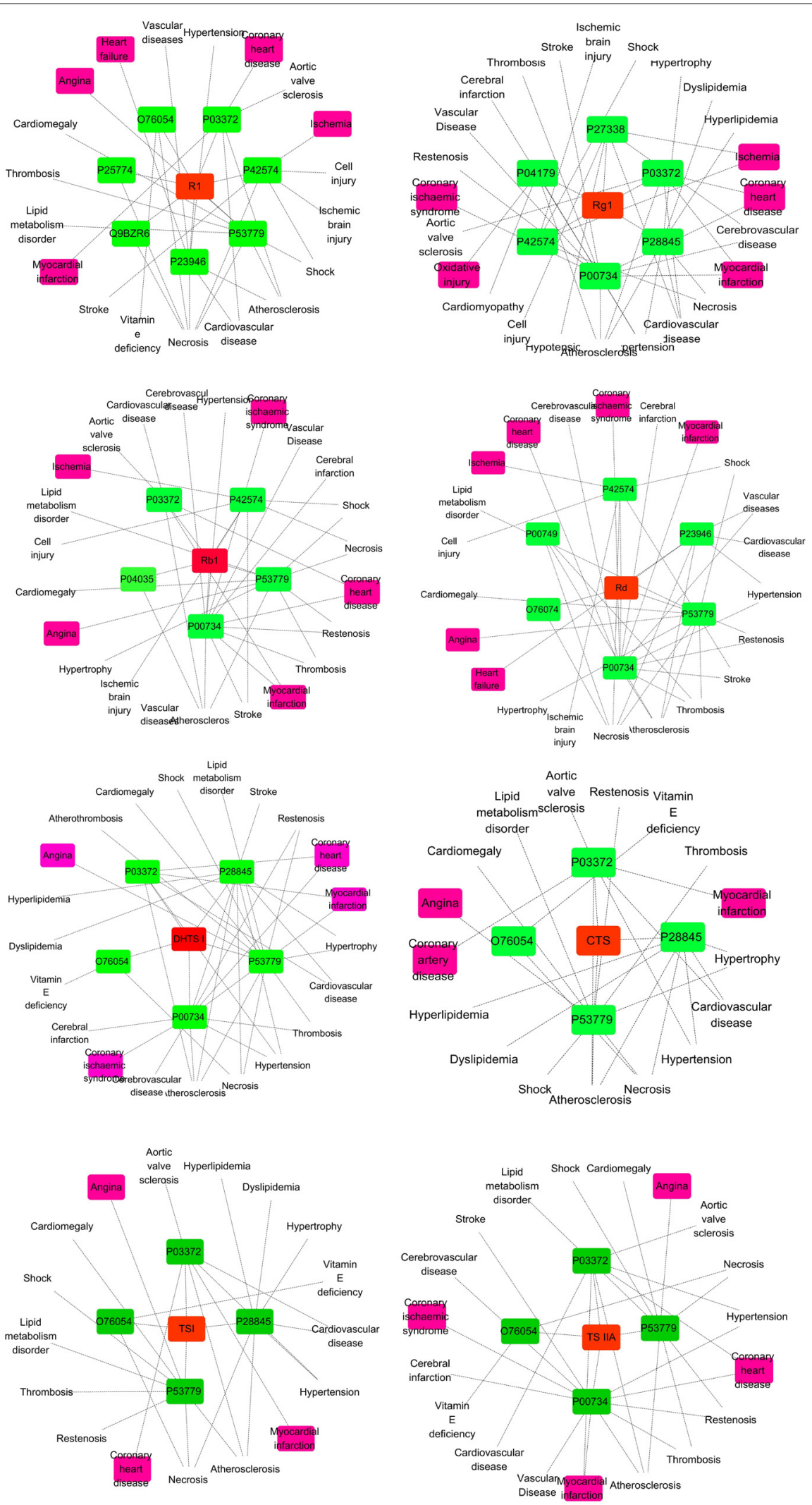

FIGURE 4 | The C-T-D network graphs of systems pharmacology for $\mathrm{R}_{1}, \mathrm{Rb}_{1}, \mathrm{Rd}, \mathrm{Rg} \mathrm{g}_{1}$, TS I, DHTS I, TS IIA, and CTS against cardiovascular affairs. The purple, green, and red rectangles represent the cardiovascular issues, target proteins, and ingredients, respectively. 


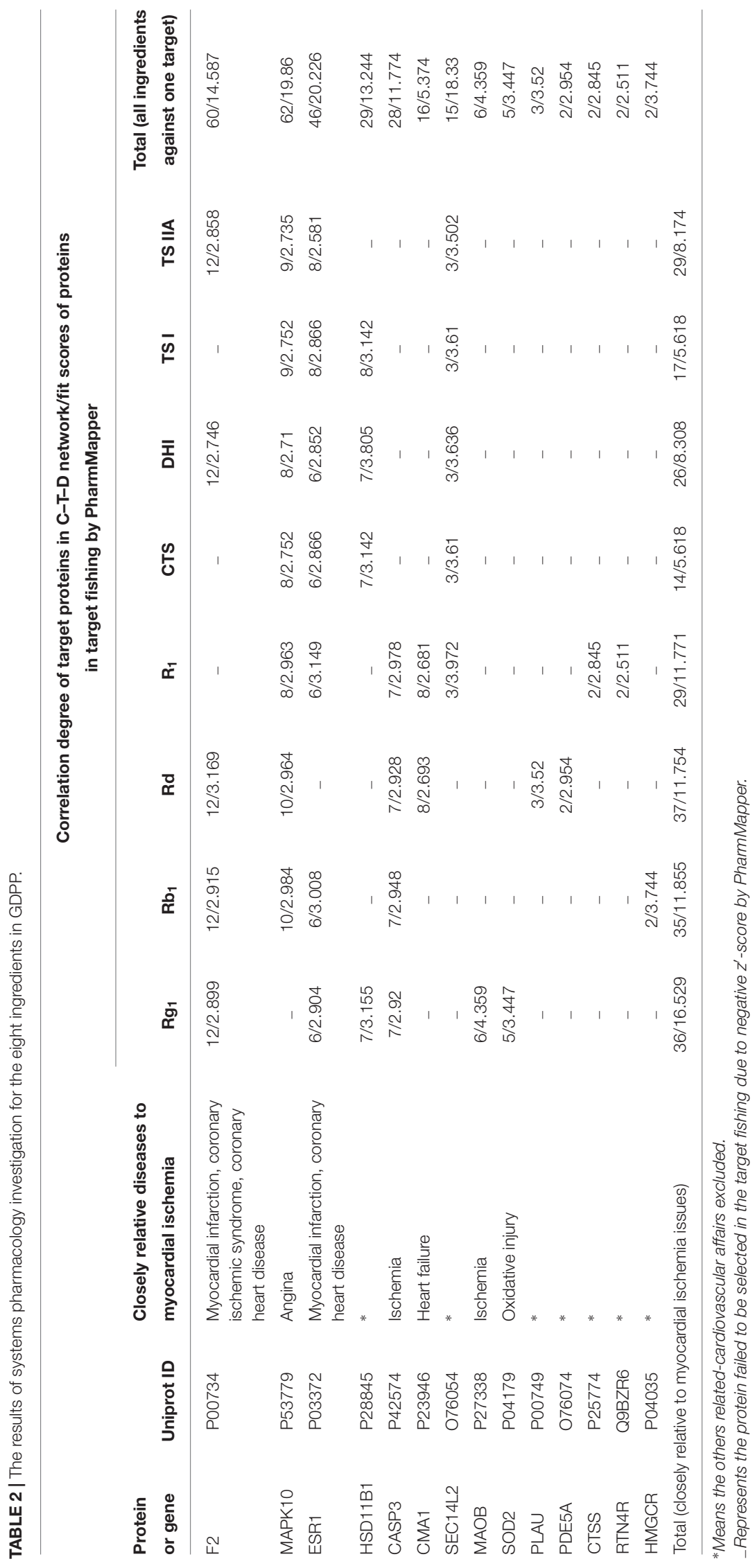




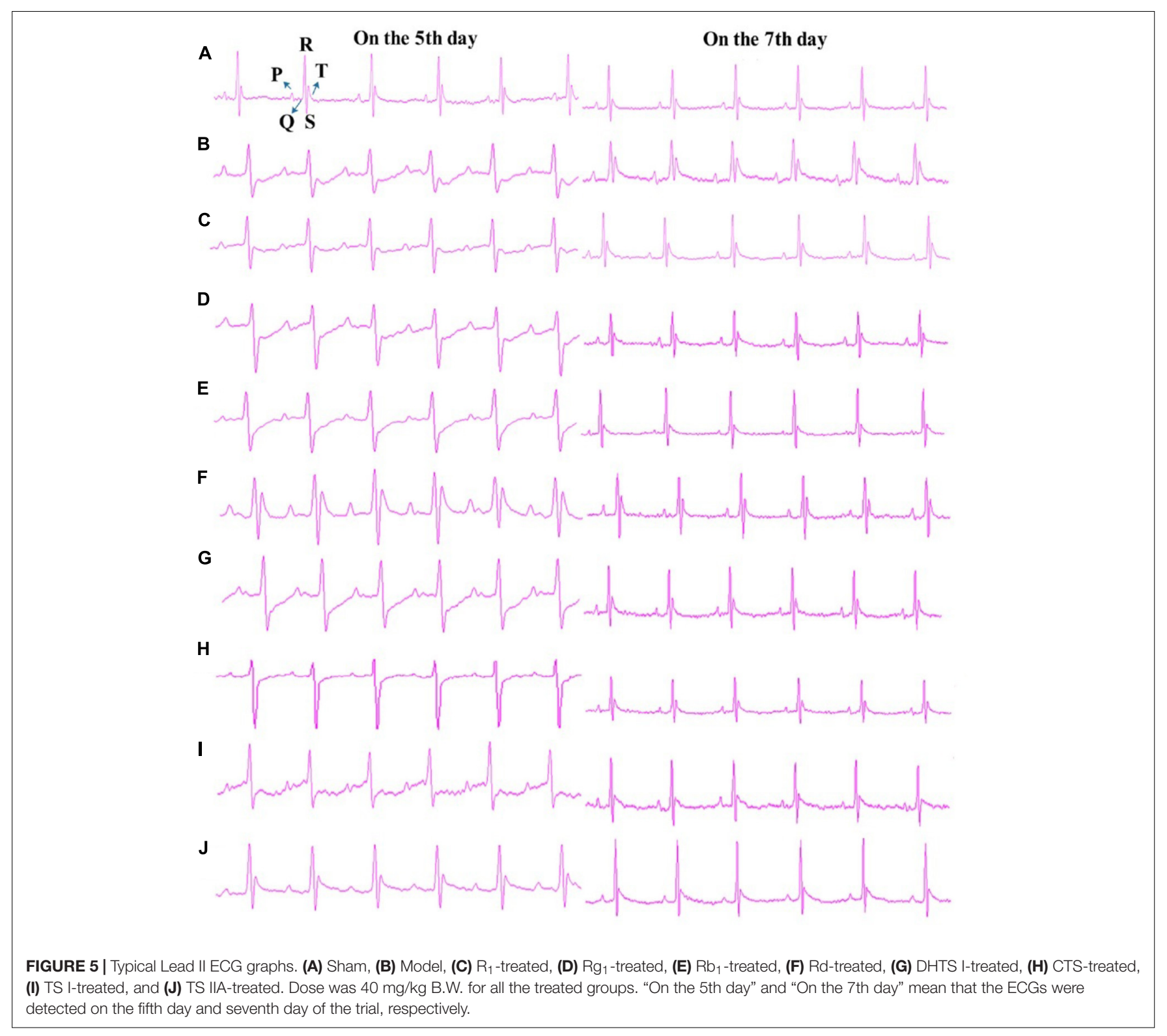

method (Yao et al., 2017b) and AUC weighting method (Lu et al., 2008). The total concentrations of the three PK markers in rat plasma were calculated by the two methods at the studied PK time points, respectively. Consequently, the integrated plasma drug concentration-time curves and PK parameters are shown in Figure 7 and Supplementary Table S5, respectively. The outlines of the PK curves by the two integrated methods were approximately similar to each other, and no apparent difference was observed for almost all the PK parameters between the two methods, except for $T_{\max }$. The AUC weighting integrated method could highlight the contribution of $\mathrm{Rb}_{1}$ to the integrated PK parameters and to the integrated PK curve appearance due to its large systematic exposure. However, the plasma drug concentration sum method could be relatively equitable to include the concentration contribution of each PK marker to the integrated PK parameters and curves at each PK time point.

\section{DISCUSSION}

Almost all the previous PK reports on herbal medicines took the constituents being adequate abundance in the herbal products and possessing favorable $\mathrm{PK}$ properties as index ones for the PK investigation (Li et al., 2007, 2008; Tong et al., 2010; Duan et al., 2011), but little further considering relative medical effect, because indeed it is unrealistic to examine the pharmacodynamic effect of all unknown and known chemicals coexisting in herbal medicines. Therefore, it would be extremely valuable to put forward and verify a general and feasible strategy for identifying representative PK markers for multicomponent herbal medicines, giving attention to both of favorable PK properties and medical effect for the identified markers. In this study, considered both the PK profiles and the therapeutic effects of the potential PK markers and highlighted the decisive role of 


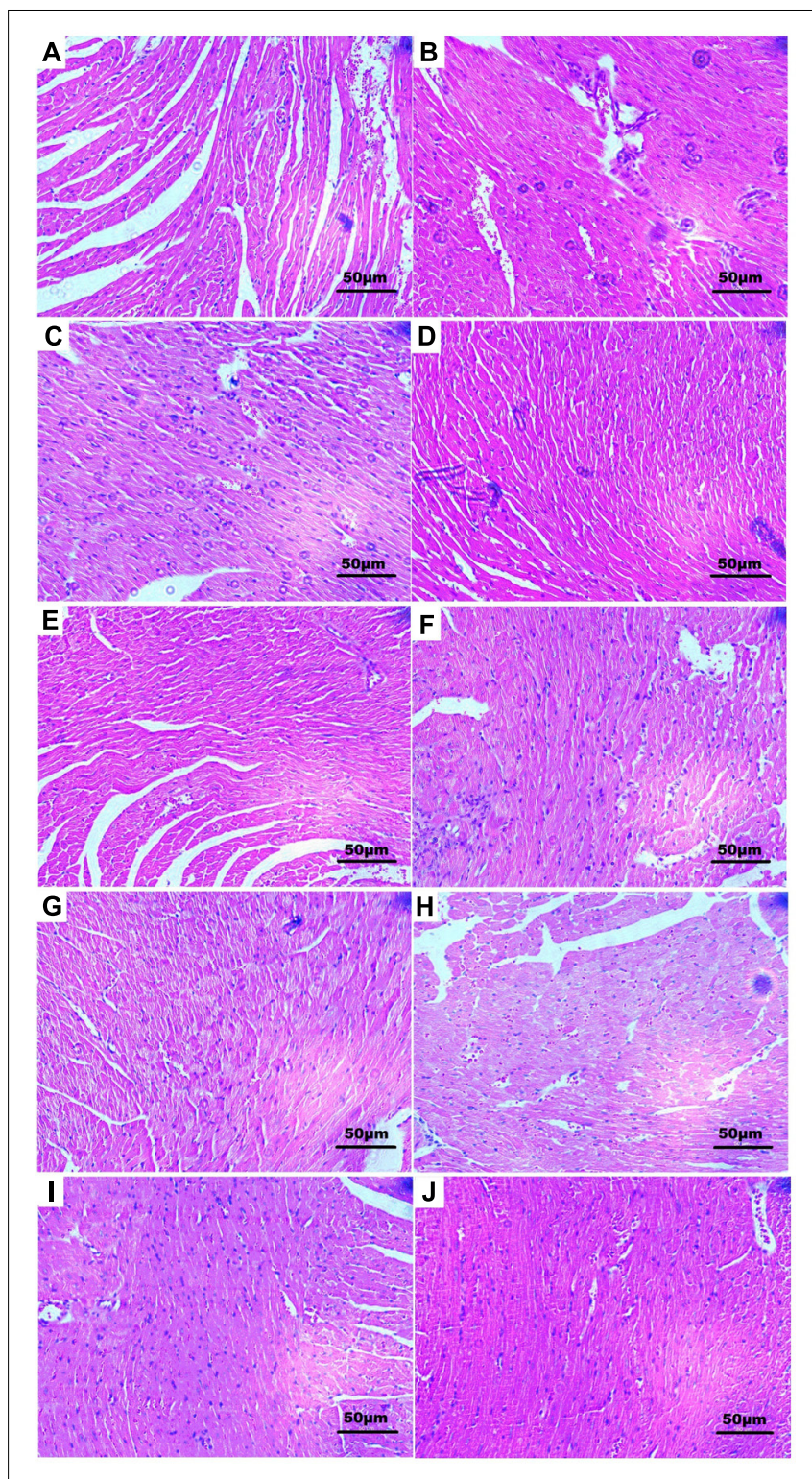

FIGURE 6 | Histological observations of hearts from each group. (A) Normal mouse showing normal architecture of the heart tissue with striations, branched appearance, and continuity with adjacent myofibrils; (B) isoprenaline-induced MI mouse showing apparent cell distortion, edema, hypochromatosis, and inferior continuity with adjacent myofibrils; (D) $\mathrm{Rg}_{1}$ and $(\mathbf{E}) \mathrm{Rb}_{1}$-treated mice showing showed less severe histological damage with mild cell distortion, edema, and good continuity with adjacent myofibrils; (I) TS I-treated mice showing myocardial lysis, and architecture disorder, and loss of striations in heart tissue; (J) TS IIA-treated group showed edema, inflammatory infiltration, and loss of striations in heart tissue. (C) $R_{1^{-}},(\mathbf{F}) \mathrm{Rd}^{-}$, (G) DHTS I-, and (H) CTS-treated mice showing extremely severe histological damage with extensive myocardial lysis and architecture wrecking. Dose was $40 \mathrm{mg} / \mathrm{kg} \mathrm{B.W}$. for all groups.

the therapeutic effects of potential PK markers with the in silico assessment, systems pharmacology, and in vivo experimental evidences. Hence, the in vivo process of the eight ingredients in GDDP was profiled. This paper is the first to report the PK profiles of the multiple ingredients of GDDP, the obtained PK parameters will be helpful to understand the relationship between administration and the therapeutic effect of GDDP, and the presented strategy will provide a reliable reference for identifying PK markers for other cardiovascular TCMs with the Danshen and Sanqi herbal pair.

As discussed in a recent review about PK study of TCMs (Shi et al., 2018), exploring or representing the whole in vivo process of a TCM is a considerable challenge owing to the extremely complex components in a TCM (Alolga et al., 2015). Danshen and Sanqi is a known herb pair contained in many cardiovascular TCM preparations, such as Fufang Danshen dripping pills, Fufang Danshen tablets, Qishenyiqi dripping pills, and Guanxin Pills (Wei et al., 2007; Yao et al., 2017a). One compound preparation containing the Danshen-Sanqi herb pair should be selected as a representative to explore the PK study strategy and represent the in vivo process of multiple-component TCMs. Successful attempt should be crucial for the PK study of a series of cardiovascular TCMs containing this herb pair. In recent years, a few efforts in PK investigation of TCMs containing the herb pair or each of them have been reported. Liu et al. (2009) studied the PK properties of multiple ingredients of Sanqi and selected $\mathrm{Ra}_{3}, \mathrm{Rb}_{1}$, and $\mathrm{Rd}$ as $\mathrm{PK}$ markers for indicating rat systemic exposure to Sanqi extract after oral administration; these markers were selected due to their easily detected blood drug concentration and high systemic exposure level (good PK properties). To identify suitable PK markers that can indicate systemic exposure to compound Danshen Pills, Lu et al. (2008) performed in vitro and in silico assessments of permeability and solubility and examined in vivo PK properties of putatively active phenolic acids, including DSS, PCAL, SAA, SAB, RA, $S A D$, and LA from the preparation. Consequently, DSS was selected as a PK marker for indicating systemic exposure to the preparation due to the relatively good PK properties of plasma DSS, poor gut permeability, and nearly undetectable levels of other ingredients in plasma and urine in dogs. In addition, Zhang et al. (2012) studied the in vivo behavior of multiple ingredients in Qishen Yiqi pills, a TCM compound preparation containing the Danshen-Sanqi pair, Huangqi (Astragalus membranaceus), and Jiangxiang. Four putatively active components, namely, DSS, $\mathrm{Rg}_{1}, \mathrm{Rb}_{1}$, and astragaloside IV, were detectable in plasma after oral administration of the preparation (1, 3, and $6 \mathrm{~g} / \mathrm{kg}$ B.W.) and selected as PK makers to profile the PK behavior of the preparation. Nevertheless, further comparison study should be carried out simultaneously to confirm the therapeutic effects of the identified PK markers.

In the present study, $\mathrm{Rg}_{1}$ and $\mathrm{Rb}_{1}$ were also detected easily, and they possessed good PK properties. However, DSS could not be detected in rat plasma at more than $15 \mathrm{~min}$ after administration of GDDP ( 0.4 and $0.8 \mathrm{~g} / \mathrm{kg}$ B.W.). This result could be attributed to that the DSS content in GDDP $(0.29 \mathrm{mg} / \mathrm{g})$ was considerably lower than that in Qishen Yiqi pills (14.52 $\mathrm{mg} / \mathrm{g}$ ). Hence, the plasma level of the absorbed DDS was below its LOD $(0.5 \mathrm{ng} / \mathrm{mL})$. Moreover, regarding the systematic exposure level, two other saponins, namely, $\mathrm{R}_{1}$ and $\mathrm{Rd}$, should be considered in our study. To exclude the unsuitable options, two points must be considered: one is that the ingredients 

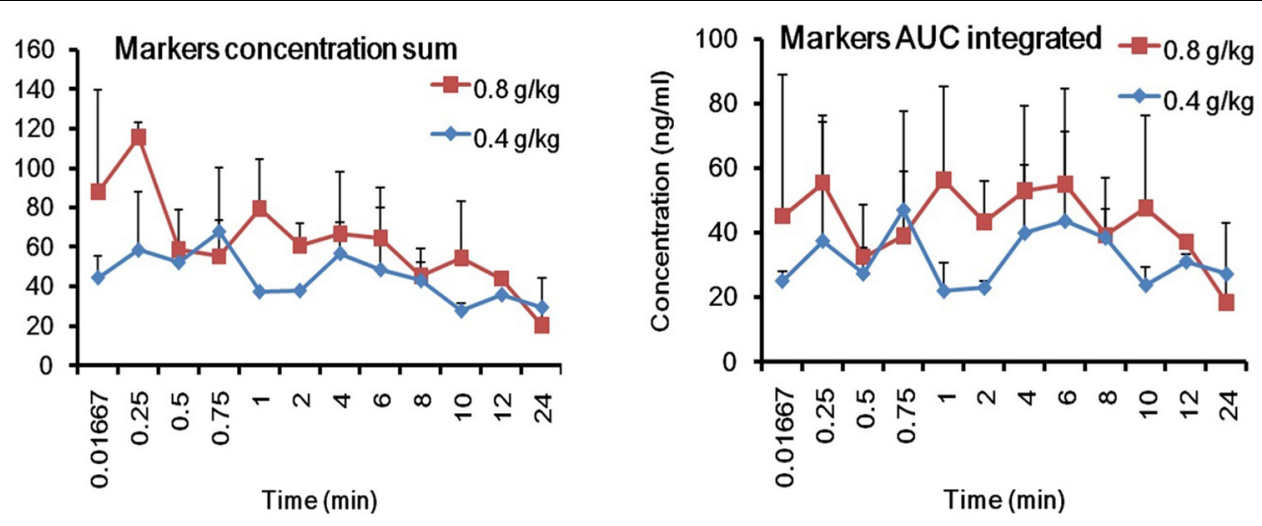

FIGURE 7 | The integrated plasma drug concentration-time curves by the plasma drug concentration sum and AUC weighing integrated methods after single p.o. administration of 0.4 and $0.8 \mathrm{~g} / \mathrm{kg}$ GDDP (mean $\pm \mathrm{SD}, n=3$ ).

should possess suitable PK profiles, and the other is that the potent therapeutic effects must be verified through comparable experimental studies about the potential PK markers. In our study, the eight ingredients showed the drawable PK curves in the present detection levels (suitable PK properties); among them, only $\mathrm{Rg}_{1}, \mathrm{Rb}_{1}$, and TS IIA showed definitely therapeutic effects against MI, which were accordant to the systems pharmacology prediction. $\mathrm{Rg}_{1}, \mathrm{Rb}_{1}$, and TS IIA should be selected as the PK markers for GDDP because the other ingredients showed no favorable therapeutic effects in our comparison study, regardless of whether they exhibited high systematic exposure level. The PK profiles of multiple ingredients were also pivotal because they confirmed the ingredients that were detectable in blood and could draw PK curves. Furthermore, the PK profiles could provide physiologically relevant basis to the possible therapeutic effects of the components of TCMs (Hao et al., 2014); these profiles are beneficial in scaling out the test objects from the 17 ingredients of GDDP to further confirm their therapeutic effects.

In TCM, four elements, that is, king-minister-assistantguide, are the basic prescription principles for the individual therapy (Li et al., 2015). Danshen and Sanqi are the main components of GDDP, and they are considered as the king and minister. Jiangxiang is considered as the assistant or guide. In our study, no other ingredient from the component herb Jiangxiang was observed in rat plasma after oral administration of GDDP ( $0.8 \mathrm{~g} / \mathrm{kg}$ B.W.). This result could be explained by that the strong liposolubility of volatile oils from Jiangxiang causes low intestinal permeability and absorption (Zhang et al., 2012). As a supposition, Jiangxiang volatile oils could facilitate the absorption of other compounds, thereby their role as the assistant or guide in the formulation.

\section{CONCLUSION}

In summary, a feasible strategy to identify PK markers for cardiovascular herbal medicines using GDDP as a case was proposed. This study considered both the PK profiles and the therapeutic effects of the potential PK markers and highlighted the decisive role of the therapeutic effects of potential PK markers with the in silico assessment, systems pharmacology, and in vivo experimental evidences. Hence, the in vivo process of the eight ingredients in GDDP was profiled. This paper is the first to report the PK profiles of the multiple ingredients of GDDP. In addition, the integrated PK parameters by the blood drug concentration sum method and AUC weighting method were calculated from the PK curves of the three markers for the first time. All the present findings will be considerably helpful to understand the relationship between administration and the therapeutic effect of GDDP. Finally, the present study will provide a reliable reference for identifying PK markers for other cardiovascular TCMs with the Danshen and Sanqi herbal pair.

\section{AUTHOR CONTRIBUTIONS}

HY conceived, designed, and performed the experiments, analyzed the data, and wrote the paper. XMH, YX, XLH, and YR performed the experiments and analyzed the data. PS conceived, designed, and performed the experiments, and analyzed the data. $\mathrm{XL}$ and LH revised the paper.

\section{FUNDING}

The authors gratefully acknowledge the financial supports of the National Nature Science Foundation of China (81202987 and 81303298), Fujian Provincial Natural Science Foundation (2018J01596 and 2016J01371), Joint Funds for the Innovation of Science and Technology, Fujian Province (2017Y9123), Training project of young talents in health system of Fujian Province (2016-ZQN-63), and training program for excellent scientific research talents of young teachers in Fujian Province University (2017).

\section{SUPPLEMENTARY MATERIAL}

The Supplementary Material for this article can be found online at: https://www.frontiersin.org/articles/10.3389/fphar.2018. 01493/full\#supplementary-material 


\section{REFERENCES}

Alolga, R. N., Fan, Y., Zhang, G., Li, J., Zhao, Y. J., Lelu Kakila, J., et al. (2015). Pharmacokinetics of a multicomponent herbal preparation in healthy Chinese and African volunteers. Sci. Rep. 5:12961. doi: 10.1038/srep12961

Bowles, S. L., Ntamo, Y., Malherbe, C. J., Kappo, A. M., Louw, J., and Muller, C. J. (2017). Intestinal transport and absorption of bioactive phenolic compounds from a chemically characterized aqueous extract of Athrixia phylicoides. J. Ethnopharmacol. 200, 45-50. doi: 10.1016/j.jep.2017.02.019

Cai, Y. F., and Dai, H. (2013). Application of guanxin danshen drop pills in patients with coronary heart disease and angina pain. China Health Ind. 25:130. doi: 10.16659/j.cnki.1672-5654.2013.25.020

Chang, Y. X., Ge, A. H., Yu, X. A., Jiao, X. C., Li, J., He, J., et al. (2016). Simultaneous determination of four phenolic acids and seven alkaloids in rat plasma after oral administration of traditional Chinese medicinal preparation Jinqi Jiangtang Tablet by LC-ESI-MS/MS. J. Pharm. Biomed. Anal. 117, 1-10. doi: $10.1016 /$ j.jpba.2015.08.030

Danhof, M. (2016). Systems pharmacology - towards the modeling of network interactions. Eur. J. Pharm. Sci. 94, 4-14. doi: 10.1016/j.ejps.2016.04.027

Duan, K. F., Yuan, Z. F., Guo, W., Meng, Y., Cui, Y., Kong, D. Z., et al. (2011). LCMS/MS determination and pharmacokinetic study of five flavone components after solvent extraction/acid hydrolysis in rat plasma after oral administration of Verbena officinalis L. extract. J. Ethnopharmacol. 135, 201-208. doi: 10.1016/ j.jep.2011.01.002

Fan, Y. Y., Man, S. L., Li, H. F., Liu, Y. X., Liu, Z., and Gao, W. Y. (2016). Analysis of bioactive components and pharmacokinetic study of herb-herb interactions in the traditional Chinese patent medicine Tongmai Yangxin Pill. J. Pharm. Biomed. Anal. 120, 364-373. doi: 10.1016/j.jpba.2015.12.032

Fang, Y., Zhang, F., Leng, Q., Wen, F., and He, L. Q. (2013). Clinical observation on treatment of coronary slow flow phenomenon with Guanxin Danshen Drop Pills. Nei Mongol. J. Tradit. Chin. Med. 13, 30-31. doi: 10.16040/j.cnki.cn151101.2013.13.113

Hao, H. P., Zheng, C. N., and Wang, G. J. (2009). Thoughts and experimental exploration on pharmacokinetic study of herbal medicines with multiplecomponents and targets. Acta Pharm. Sin. 44, 270-275. doi: 10.16438/j.05134870.2009 .03 .002

Hao, H. P., Zheng, X., and Wang, G. J. (2014). Insights into drug discovery from natural medicines using reverse pharmacokinetics. Trends Pharmacol. Sci. 35, 168-177. doi: 10.1016/j.tips.2014.02.001

Hao, P. P., Jiang, F., Cheng, J., Ma, L. Y., Zhang, Y., and Zhao, Y. X. (2017). Traditional Chinese medicine for cardiovascular disease. J. Am. Coll. Cardiol. 69, 2952-2966. doi: 10.1016/j.jacc.2017.04.041

Hara, Y. (2011). Tea catechins and their applications as supplements and pharmaceutics. Pharm. Res. 64, 100-104. doi: 10.1016/j.phrs.2011.03.018

Hu, T., To, K. K., Wang, L., Zhang, L., Lu, L., Shen, J., et al. (2014). Reversal of P-glycoprotein (P-gp) mediated multidrug resistance in colon cancer cells by cryptotanshinone and dihydrotanshinone of Salvia miltiorrhiza. Phytomedicine 21, 1264-1272. doi: 10.1016/j.phymed.2014.06.013

Li, W., Li, Z. W., Han, J. P., Li, X. X., Ga, J., and Liu, C. X. (2008). Determination and pharmacokinetics of danshensu in rat plasma after oral administration of danshen extract using liquid chromatography/tandem mass spectrometry. Eur. J. Drug Metab. Pharmacokinet. 33, 9-16. doi: 10.1007/BF03191013

Li, X. Y., Sun, J. G., Wang, G. J., Hao, H. P., Liang, Y., Zheng, Y. T., et al. (2007). Simultaneous determination of panax notoginsenoside R1, ginsenoside $\mathrm{Rg} 1, \mathrm{Rd}, \mathrm{Re}$ and $\mathrm{Rb} 1$ in rat plasma by HPLC/ESI/MS: platform for the pharmacokinetic evaluation of total panax notoginsenoside, a typical kind of multiple constituent traditional Chinese medicine. Biomed. Chromatogr. 2007, 735-746. doi: 10.1002/bmc.813

Li, Y. Z., Wang, Y. L., Tai, W., Yang, L., Chen, Y., Chen, C. Q., et al. (2015). Challenges and solutions of pharmacokinetics for efficacy and safety of traditional Chinese medicine. Curr. Drug Metab. 16, 765-776. doi: 10.2174/ 138920021609151201114223

Lipinski, C. A., Lombardo, F., Dominy, B. W., and Feeney, P. J. (2001). Experimental and computational approaches to estimate solubility and permeability in drug discovery and development settings. Adv. Drug Deliv. Rev. 46, 3-26. doi: 10.1016/S0169-409X(00)00129-0

Liu, H. F., Yang, J. L., Du, F. F., Gao, X. M., Ma, X. T., Huang, Y. H., et al. (2009). Absorption and disposition of ginsenosides after oral administration of Panax notoginseng extract to rats. Drug Metab. Disp. 37, 2290-2298. doi: $10.1124 / \mathrm{dmd} .109 .029819$

Liu, X., Ouyang, S., Yu, B., Liu, Y., Huang, K., Gong, J., et al. (2010). Pharmmapper server: a web server for potential drug target identification via pharmacophore mapping approach. Nucleic Acids Res. 38, W609-W614. doi: 10.1093/nar/ gkq300

Lu, L., Sun, X., Chen, C., Qin, Y., and Guo, X. (2018). Shexiang baoxin pill, derived from the traditional chinese medicine, provides protective roles against cardiovascular diseases. Front. Pharmacol. 9:1161. doi: 10.3389/fphar.2018. 01161

Lu, T., Yang, J. L., Gao, X. M., Chen, P., Du, F. F., Sun, Y., et al. (2008). Plasma and urinary tanshinol from Salvia miltiorrhiza (Danshen) can be used as pharmacokinetic markers for cardiotonic pills, a cardiovascular herbal medicine. Drug Metab. Dispos. 36, 1578-1586. doi: 10.1124/dmd.108. 021592

Miller, L. H., and Su, X. (2011). Artemisinin: discovery from the Chinese herbal garden. Cell 146, 855-858. doi: 10.1016/j.cell.2011.08.024

Shi, P. Y., Lin, X. H., and Yao, H. (2015). Pharmacokinetics of isoorientin, a natural active ingredient, in Sprague-Dawley male rats after oral and intravenous administration. Xenobiotica 45, 999-1008. doi: 10.3109/00498254. 2015.1028513

Shi, P. Y., Lin, X. H., and Yao, H. (2018). A comprehensive review of recent studies on pharmacokinetics of traditional Chinese medicines (2014-2017) and perspectives. Drug Meta. Rev. 50, 161-192. doi: 10.1080/03602532.2017. 1417424

Smith, S. C. Jr., Collins, A., Ferrari, R., Holmes, D. R. Jr., Logstrup, S., McGhie, D. V., et al. (2012). Our time: a call to save preventable death from cardiovascular disease (heart disease and stroke). J. Am. Coll. Cardiol. 60, 2343-2348. doi: 10.1016/j.jacc.2012.08.962

Stone, R. (2008). Lifting the veil on traditional Chinese medicine. Science 319, 709-710. doi: 10.1126/science.319.5864.709

Tang, S. M., Xing, X. Y., Deng, X. H., Zhao, N. N., Li, G., Yang, R. C., et al. (2016). Research progress of Guanxin Danshen formula and its effective components in treating coronary artery heart disease. China J. Chin. Mater. Med. 41, 3721-3726. doi: 10.4268/cjcmm20162004

Tong, L., Wan, M. X., Zhou, D. D., Gao, J., Zhu, Y. H., and Bi, K. S. (2010). LC-MS/MS determination and pharmacokinetic study of albiflorin and paeoniflorin in rat plasma after oral administration of Radix Paeoniae Alba extract and Tang-Min-Ling-Wan. Biomed. Chromatogr. 24, 1324-1331. doi: 10.1002/bmc. 1443

Wang, X., Pan, C., Gong, J., Liu, X., and Li, H. (2016). Enhancing the enrichment of pharmacophore-based target prediction for the polypharmacological profiles of drugs. J. Chem. Inf. Model. 56, 1175-1183. doi: 10.1021/acs.jcim.5b00690

Wang, X., Shen, Y., Wang, S., Li, S., Zhang, W., Liu, X., et al. (2017). Pharm Mapper 2017 update: a web server for potential drug target identification with a comprehensive target pharmacophore database. Nucleic Acids Res. 45, W356-W360. doi: 10.1093/nar/gkx374

Wei, Y. J., Qi, L. W., Li, P., Luo, H. W., Yi, L., and Sheng, L. H. (2007). Improved quality control method for Fufang Danshen preparations through simultaneous determination of phenolic acids, saponins and diterpenoid quinones by HPLC coupled with diode array and evaporative light scattering detectors. J. Pharm. Biomed. Anal. 45, 775-784. doi: 10.1016/j.jpba.2007.07.013

Xue, T. H., and Roy, R. (2003). Studying traditional Chinese medicine. Science 300, 740-741. doi: 10.1126/science.300.5620.740

Yang, S., Wang, B. L., Xia, X. J., Li, X., Wang, R. Y., Sheng, L., et al. (2015). Simultaneous quantification of three active alkaloids from a traditional Chinese medicine Ramulus Mori (Sangzhi) in rat plasma using liquid chromatographytandem mass spectromety. J. Pharm. Biomed. Anal. 109, 177-183. doi: 10.1016/ j.jpba.2015.02.019

Yang, S. F., Shi, P. Y., Huang, X. M., Zhao, M. F., Li, S. G., Wu, Y. J., et al. (2016). Pharmacokinetics, tissue distribution and protein binding studies of chrysocauloflavone I in rats. Planta Med. 82, 217-223. doi: 10.1055/s-00351558159

Yao, H., Huang, X. M., Li, S. G., Wu, Y. J., Lin, X. H., and Shi, P. Y. (2017a). Simultaneous determination of eight phenolic acids, five saponins and four tanshinones for quality control of compound preparations containing Danshen-Sanqi herb-pair by HPLC-DAD. Pharmacogn. Mag. 13, 64-75. doi: $10.4103 / 0973-1296.197651$ 
Yao, H., Shi, P. Y., Huang, X. M., Shen, Z. H., Su, Y., Yang, C. L., et al. (2017b). A strategy for integrated pharmacokinetic study of cardiovascular herbal medicines based on chemiluminescence and HPLC-MS/MS assays: a case using Danshen injection. RSC Adv. 7, 13570-13583. doi: 10.1039/c7ra00001d

Zhang, J. X., Li, Y., Chen, S. S., Zhang, L. L., Wang, J. H., Yang, Y. F., et al. (2015). Systems pharmacology dissection of the anti-inflammatory mechanism for the medicinal herb Folium Eriobotryae. Int. J. Mol. Sci. 16, 2913-2941. doi: 10.3390/ijms16022913

Zhang, Y. F., Shi, P. Y., Yao, H., Shao, Q., and Fan, X. H. (2012). Metabolite profiling and pharmacokinetics of herbal compounds following oral administration of a cardiovascular multi-herb medicine (Qishen Yiqi Pills) in rats. Curr. Drug Metab. 13, 510-523. doi: 10.2174/1389200211209050510

Zheng, C. L., Pei, T. L., Huang, C., Chen, X. T., Bai, Y. F., Xue, J., et al. (2016). A novel systems pharmacology platform to dissect action mechanisms of traditional Chinese medicines for bovine viral diarrhea disease. Eur. J. Pharm. Sci. 94, 33-45. doi: 10.1016/j.ejps.2016.05.018

Zhou, W., Tam, K. Y., Meng, M. X., Shan, J. J., Wang, S. C., Ju, W. Z., et al. (2015). Pharmacokinetics screening for multi-components absorbed in the rat plasma after oral administration of traditional Chinese medicine Flos Lonicerae Japonicae-Fructus Forsythiae herb couple by sequential negative and positive ionization ultra-high-performance liquid chromatography/tandem triple quadrupole mass spectrometric detection. J. Chromatogr. A 1376, 84-97. doi: 10.1016/j.chroma.2014. 12.018

Conflict of Interest Statement: The authors declare that the research was conducted in the absence of any commercial or financial relationships that could be construed as a potential conflict of interest.

Copyright (c) 2018 Yao, Huang, Xie, Huang, Ruan, Lin, Huang and Shi. This is an open-access article distributed under the terms of the Creative Commons Attribution License (CC BY). The use, distribution or reproduction in other forums is permitted, provided the original author(s) and the copyright owner(s) are credited and that the original publication in this journal is cited, in accordance with accepted academic practice. No use, distribution or reproduction is permitted which does not comply with these terms. 